\title{
Layer-by-Layer Technique to Developing Functional Nanolaminate Films with Antifungal Activity
}

\author{
María José Fabra $^{1}$ - Maria L. Flores-López ${ }^{2} \cdot$ Miguel A. Cerqueira ${ }^{2}$. \\ Diana Jasso de Rodriguez ${ }^{3}$. Jose M. Lagaron ${ }^{1}$ • António A. Vicente ${ }^{2}$
}

Received: 19 June 2015 / Accepted: 9 November 2015 /Published online: 16 November 2015

(C) Springer Science+Business Media New York 2015

\begin{abstract}
The layer-by-layer (LbL) deposition method was used to build up alternating layers (five) of different polyelectrolyte solutions (alginate, zein-carvacrol nanocapsules, chitosan and chitosan-carvacrol emulsions) on an aminolysed/ charged polyethylene terephthalate (A/C PET) film. These nanolaminated films were characterised by contact angle measurements and through the determination of water vapour (WVTR) and oxygen $\left(\mathrm{O}_{2} \mathrm{TR}\right)$ transmission rates. The effect of active nanolaminated films against the Alternaria sp. and Rhizopus stolonifer was also evaluated. This procedure allowed developing optically transparent nanolaminated films with tuneable water vapour and gas properties and antifungal activity. The water and oxygen transmission rate values for the multilayer films were lower than those previously reported for the neat alginate or chitosan films. The presence of carvacrol and zein nanocapsules significantly decreased the water transmission rate (up to $40 \%$ ) of the nanolaminated films. However, the $\mathrm{O}_{2}$ TR behaved differently and was only improved (up to $45 \%$ ) when carvacrol was encapsulated, i.e. nanolaminated films prepared by alternating alginate with nanocapsules of zein-carvacrol layers showed better oxygen
\end{abstract}

María José Fabra

mjfabra@iata.csic.es

Miguel A. Cerqueira

miguelcerqueira@deb.uminho.pt

1 Novel Materials and Nanotechnology Group, IATA-CSIC, Avda. Agustin Escardino 7, 46980 Paterna, Valencia, Spain

2 Centre of Biological Engineering, Universidade do Minho, Campus de Gualtar, 4710-057 Guimaraes, Braga, Portugal

3 Universidad Autonoma Agraria Antonio Narro, Calzada Antonio Narro No. 1923, Colonia Buenavista, 25315 Saltillo, Coahuila, México barrier properties than those prepared as an emulsion of chitosan and carvacrol. These films containing zein-carvacrol nanocapsules also showed the highest antifungal activity ( $30 \%$ ), which did not significantly differ from those obtained with the highest amount of carvacrol, probably due to the controlled release of the active agent (carvacrol) from the zeincarvacrol nanocapsules. Thus, this work shows that nanolaminated films prepared with alternating layers of alginate and zein-carvacrol nanocapsules can be considered to improve the shelf-life of foodstuffs.

Keywords Carvacrol $\cdot$ Alginate $\cdot$ Chitosan $\cdot$ Nanocapsules · Barrier properties

\section{Introduction}

Nanotechnology provides food scientists a great number of ways to create novel laminate films suitable for use in the food industry. A nanolaminate consists of two or more layers of material with nanometre dimensions that are physically or chemically bonded to each other. One of the most powerful methods is based on the layer-by-layer (LbL) deposition technique, in which the charged surfaces are coated with interfacial films consisting of multiple nanolaminated films of different materials (Decher and Schlenoff 2003, Weis, Takhiston and McClements 2006).

The sequential build-up of polymers via the LbL technique provides an efficient and versatile means for depositing functional polymer coatings on surfaces (Pinheiro et al. 2012, Weiss et al. 2006). Thus, a variety of functional thin films can be produced using the LbL assembly technique. Thin films, typically $<1 \mu \mathrm{m}$ thick, are created by alternately exposing a substrate to positively and negatively charged molecules or particles. Each individual layer may be $1-100 \mathrm{~nm}$ thick 
depending on the linear charge density and molecular weight of the adsorbing polymers, extent of film hydration and ionic strength, temperature, deposition time, counter ion and $\mathrm{pH}$ of the species being deposited (Zhong, Li and Haynie 2006). Some advantages when these coatings are at the nano-scale are high stability on the substrate surface, facility of preparation (Peng et al. 2001) and lower concentration of materials required (Hinrichsen et al. 2003).

One of the most used polysaccharides in multilayer construction is chitosan, which is a very interesting polymer for numerous applications due to its biocompatibility, biodegradability and nontoxicity, as well as its antimicrobial activity. As a cationic polymer, chitosan may be associated with anionic polyelectrolytes such as alginate, leading to the formation of a polyelectrolyte multilayer. Alginate is a linear block of copolymers composed of varying proportions of 1,4-linked $\beta$-D mannuronic acid (M) and $\alpha$-Lguluronic acid $(G)$, forming regions of M-blocks, Gblocks and blocks of alternating sequence (MG-blocks). They have the ability to form gels in the presence of multivalent counterions, which is a direct consequence of the fact that alginates are polyelectrolytes.

In addition, $\mathrm{LbL}$ technique could be used to encapsulate various substances (hydrophilic, amphiphilic or lipophilic) within the nanolaminated films by incorporating them, for example, in oil droplets or association colloids (such as micelles or liposomes). Thus, it would be possible to incorporate active functional agents such as antimicrobials, antibrowning agents, antioxidants, enzymes, flavours and colours into the films. These functional agents would increase the shelf-life and quality of coated foods (Weiss et al. 2006).

Essential oils show a great potential to be used as antimicrobial compounds both when directly added to foods and via their use in the form of vapour. Many essential oils, which are hydrophobic and volatile compounds derived from plants, have shown natural antifungal, insecticidal, antimicrobial and antioxidant properties. The inhibitory effect of carvacrol on the growth of various microorganism is well documented and described extensively (Ben Arfa, Combes, PreziosiBelloy, Gontard and Challer 2006; Burt 2004, Nostro and Papalia 2012). In fact, Zabka and Pavela (2013) have recently been demonstrated that carvacrol is one of the most effective antifungal essential oil. Carvacrol is classified as generally recognized as safe (GRAS), being one of the main components of thyme spice, by the US Food and Drug Administration (FDA: Lambert et al. 2001). Carvacrol might be incorporated within biopolymer materials and the efficiency of this system is determined by its (controlled) diffusion and release properties and if concentrations are kept high enough for the desired antimicrobial impact. However, the inherent volatility of essential oils could imply that the amount of carvacrol required to reach the inhibitory effect will be higher. Thus, nanoencapsulation can be applied to avoid this problem, favouring a controlled release of the active compound which could prolong the antimicrobial effect during storage.

Zein, a corn prolamine protein, is a GRAS food-grade ingredient. It has three quarters of lipophilic and one quarter of hydrophilic amino acid residues. Because of its high hydrophobicity, zein has been studied in food and pharmaceutical industries for encapsulation and sustained release of hydrophobic bioactives, such as fish oil (Zhong et al. 2009), $\alpha$ tocopherol (Luo et al. 2011), vitamin D, curcumin (Patel, Hu, Tiwari, and Velikov 2010) and thymol (Zhang et al. 2014).

In a previous work, Carneiro-da-Cunha et al. (2010) reported the ability of chitosan and alginate polyelectrolyte solutions to form nanolaminate films. The present work is a follow-up of that study, where results were presented on the physical and thermal properties of a chitosan/alginate nanolaminated PET film. The aim of the present work is to analyse the effect of adding carvacrol on antifungal properties of nanolaminated films assembled through LbL technique. The effect of encapsulating carvacrol into zein nanocapsules was also compared with those obtained by direct addition into a chitosan matrix. Thus, nanolaminated films with sodium alginate and aqueous solutions of zein nanocapsules containing carvacrol were also obtained by LbL assembly and characterized.

\section{Materials and Methods}

\section{Materials}

Films of polyethylene terephthalate (PET) were obtained from Canson (France). Sodium alginate was obtained from Manutex RSX (Kelco International, Ltd., Portugal) and chitosan $(91.23 \%$ deacetylation degree and high molecular weight) was purchased from Golden-Shell Biochemical Co., Ltd. (Zhejiang, China). The 1,6-hexanediamine ( $>98 \%$ of purity) and carvacrol ( $>95.5 \%$ of purity) were purchased from Sigma-Aldrich (Germany). Lactic acid with $90 \%$ of purity and hydrochloric acid with $37 \%$ of purity were obtained from Sigma-Aldrich (Germany). Glycerol was supplied by Panreac (Spain). Purified zein and Tween 80 were purchased from Acros Organics (Belgium). Ethanol ( $>99.8 \%$ of purity) and sodium hydroxide were obtained from Riedel-de Haën (Germany).

\section{Preparation of Nanolaminated Film Assembly}

The nanolaminated films were built on aminolysed/charged PET support films. Five alternative polysaccharide (alginate and chitosan) or zein/carvacrol nanocapsules were adsorbed onto the support with the sequence described below (Table 1). 
Table 1 Description of the five successive layers of each multilayer system

\begin{tabular}{lllllll}
\hline Nanolaminated film & Support film & 1st layer & 2nd layer & 3rd layer & 4th layer & 5th layer \\
\hline A & A/C PET & \multirow{2}{*}{ Alg } & $\mathrm{CH}$ & Alg & $\mathrm{CH}$ & Alg \\
B & & & $\mathrm{Z} / \mathrm{C}$ & & $\mathrm{CH}$ & \\
C & & $\mathrm{Z} / \mathrm{C}$ & & $\mathrm{Z} / \mathrm{C}$ & \\
D & & $\mathrm{CH}-0.09 \mathrm{C}^{\mathrm{a}}$ & & $\mathrm{CH}-0.09 \mathrm{C}^{\mathrm{a}}$ & \\
E & & & $\mathrm{CH}-0.6 \mathrm{C}^{\mathrm{a}}$ & & $\mathrm{CH}-0.6 \mathrm{C}^{\mathrm{a}}$ & \\
\hline
\end{tabular}

A/C PET aminolysed/charged PET, Z/C nanocapsules zein-carvacrol, $A l g$ alginate, $\mathrm{CH}$ chitosan, $\mathrm{C}$ carvacrol

${ }^{a}$ Carvacrol concentration in the $\mathrm{CH}$ emulsion, 0.09 and $0.6 \%$, respectively

\section{Aminolysis of Polyethylene Terepthalate Surface}

Polyethylene terephthalate (PET) films were cut into rectangular pieces of $0.8 \times 5.0 \mathrm{~cm}$ and circular pieces of $5.0 \mathrm{~cm}$ of diameter and were aminolysed as described by $\mathrm{Fu}$ et al. (2005). Briefly, PET films were cleaned in ethanol/water $(1: 1, v / v)$ solution for $3 \mathrm{~h}$, followed by a thorough rinsing with distilled water, and dried at $30{ }^{\circ} \mathrm{C}$ for $24 \mathrm{~h}$. Afterwards, the films were immersed into $0.06 \mathrm{~g} \mathrm{~mL}^{-1} 1,6$-hexanediamine/ propanol solution at $37^{\circ} \mathrm{C}$ for $4 \mathrm{~h}$, thoroughly washed with distilled water to remove free 1,6-hexadiamine and finally dried at $37{ }^{\circ} \mathrm{C}$ for $24 \mathrm{~h}$. The aminolysed PET films were treated with $0.1 \mathrm{HCl}$ solution for $3 \mathrm{~h}$ at room temperature $\left(20^{\circ} \mathrm{C}\right)$, and then washed with large amount of distilled water, dried at $30^{\circ} \mathrm{C}$ for $24 \mathrm{~h}$ and termed aminolysed/charged PET (A/C PET). This procedure was needed to positively charge the otherwise natural PET surface, aiming at a stronger interaction with the negatively charged alginate.

\section{Preparation of Polyelectrolyte Solutions}

Sodium alginate solution was prepared dissolving $0.2 \%(w / v)$ sodium alginate in distilled water and stirred, at room temperature, until sodium alginate was completely dissolved. Based on screening studies related to the spreading coefficient of these alginate solutions onto food surfaces (data not shown), glycerol at $0.05 \%(w / v)$ and Tween 80 at $0.05 \%(w / v)$ were added as a plasticizer and as a surfactant, respectively. This aspect was considered because the affinity between the food surface and coating formulation is fundamental in the coating design, considering that the effective spreading of a coating on a food is greatly influenced by the wettability of the surface by the coating formulation. The $\mathrm{pH}$ of the film forming solution was adjusted to 7.0 with a solution of $1 \mathrm{~mol} \mathrm{~L}^{-1}$ sodium hydroxide.

Chitosan $(0.6 \% w / v)$ solution was dispersed in an aqueous solution of lactic acid $(1.0 \% \mathrm{v} / \mathrm{v})$ under magnetic stirring until it was completely dissolved at room temperature. Based on the abovementioned screening studies, glycerol at $0.1 \%(w / v)$ and Tween 80 at $0.1 \%(w / v)$ were added as plasticizer and surfactant, respectively. Finally, chitosan solution was adjusted to $\mathrm{pH} 3.0$ with a $1 \mathrm{~mol} \mathrm{~L}^{-1}$ lactic acid solution.
Chitosan-carvacrol (CH-C) solutions were prepared by adding the active compound to chitosan solutions to reach a final concentration of 0.09 or $0.6 \%(w / v)$. CH-C mixtures were emulsified at room temperature using a rotor-stator homogenizer (Ultraturrax, Jankeand Kunkel, Staufen, Germany) at 10,000 rpm for $2 \mathrm{~min}$. Solution nomenclature was CH-nC, being the $n$ value the carvacrol concentration in the chitosan solution.

To clarify, the carvacrol essential oil concentrations used were selected according to, on the one hand, the encapsulation efficiency of zein-carvacrol systems $(0.09 \%, w / v)$ and, on the other hand, the amount of chitosan used in the solution $(0.6 \%$, $w / v)$ in order to obtain a chitosan/carvacrol ratio of 1:1.

Zein-Nanocapsules Development Solutions of zeincarvacrol nanocapsules were prepared as follows: zein $\left(2 \mathrm{mg} \mathrm{mL}^{-1}\right)$ was dissolved in ethanol/water binary solvent $(75: 25 v / v)$ and carvacrol was added to zein solution at a zeincarvacrol ratio of 1:0.5. After stirring overnight, zeincarvacrol nanocapsules were obtained by pumping $10 \mathrm{~mL}$ of zein-carvacrol solution into $50 \mathrm{~mL}$ of distilled water which was continuously stirred at $600 \mathrm{rpm}$. Finally, aqueous solution of zein-carvacrol nanocapsules was adjusted to $\mathrm{pH} 3.0$ with $1 \mathrm{~mol} \mathrm{~L}^{-1}$ lactic acid solution.

\section{Coating Procedure of A/C PET}

A/C PET pieces were immersed into the alginate solution for $20 \mathrm{~min}$ and subsequently rinsed with deionized water with the same $\mathrm{pH}(7.0)$. The samples were dried by hanging them inside a chamber with a nitrogen flow in order to speed up the process. This procedure was repeated with the other polyelectrolyte solutions (chitosan, chitosan/carvacrol) and charged nanocapsules' suspensions based on zein/carvacrol) but, in this case, samples were rinsed with an aqueous solution at $\mathrm{pH}$ 3.0. This procedure was repeated with the alternate deposition of a total of five nanolayers with different sequences, as described in Table 1. As a result, five types of nanolaminated coatings on A/C PET films were produced and maintained at $20 \pm 2{ }^{\circ} \mathrm{C}$ and $50 \pm 5 \%$ of relative humidity $(\mathrm{RH})$ provided by the laboratory air-conditioning system before analysis. 


\section{Physicochemical Characterization}

\section{Zeta Potential and Particle Size}

The zeta potential and the particle size of each polyelectrolyte solution were determined by dynamic light scattering (DLS) (Zetasizer Nano ZS, Malvern Instruments, UK). Each sample was analysed in a folded capillary cell. Three replicates were conducted.

\section{Contact Angle Measurements}

Contact angles $\left(\theta^{\circ}\right)$ of the original PET, A/C PET and the subsequent nanolaminated coatings' surface were measured by the sessile drop method (Newman and Kwok 1999), in which a droplet of ultra-pure water was placed on a horizontal surface using a contact angle meter (OCA 20, Dataphysics, Germany), equipped with an image analysis software. The water droplet $(2 \mu \mathrm{L})$ was placed on the horizontal surface with a $500-\mu \mathrm{L}$ syringe (Hamilton, Switzerland) with a needle of $0.75-\mathrm{mm}$ diameter. Measurements were made at 0, 15 and $30 \mathrm{~s}$ and, for each type of surface, three film samples were used. For each film sample, seven contact angle measurements were carried out at $20.5 \pm 0.3{ }^{\circ} \mathrm{C}$.

\section{Encapsulation Efficiency}

Encapsulation efficiency $(E E \%)$ was determined after the separation of the zein-carvacrol nanocapsules from the supernatant with free carvacrol. The separation was performed using an Amicon ${ }^{\circledR}$ Ultra-0.5 centrifugal filter device (Amicon ${ }^{\circledR}$ Ultra0.5-mL 3K device, Millipore Corp., Ireland). Briefly, $0.5 \mathrm{~mL}$ of sample was added to the Amicon ${ }^{\circledR}$ and was centrifuged at $14,000 \times g$ during $10 \mathrm{~min}$. After centrifugation, a filtrate with free carvacrol and a concentrate with zein-carvacrol nanocapsules were obtained. The filtrate was then assayed spectrophotometrically at $271 \mathrm{~nm}$ and the amount of free vitamin was determined. $E E \%$ was determined as follows:

$E E \%=\frac{[\text { Carvacrol }]_{\text {total }}-[\text { Carvacrol }]_{\text {free }}}{[\text { Carvacrol }]_{\text {total }}} \cdot 100$

where [Carvacrol $]_{\text {total }}$ represents the total amount of carvacrol added during nanoparticles production and [Carvacrol $]_{\text {free }}$ the free carvacrol in the filtrate.

\section{Barrier Properties}

Water Vapour Transmission Rate The water vapour transmission rate (WVTR) of the nanolaminated films was measured according to the ASTM E96 (ASTM 2011) gravimetric method (Casariego et al. 2009; McHugh et al. 1993). Measurements were taken in triplicate. WVP was determined experimentally for the sustaining layer (A/C PET) and for the obtained nanolaminated films (multilayers A, B, C, D and E). The WVTR of the polysaccharide or polysaccharide/protein nanolaminated coating (without A/C PET) was determined by the following equation (Cooksey, Marsh and Doar 1999).

$\mathrm{WVTR}_{B}=\frac{1}{\frac{1}{\mathrm{WVTR}_{T}}-\frac{1}{\mathrm{WVTR}_{A}}}$

where A, B and T refer to the sustaining layer (A/C PET), the polysaccharide-protein nanolaminated coating and the resulting nanolaminated film (A, B, C, D and E), respectively.

Oxygen Transmission Rate Oxygen transmission rate was determined based on ASTM (2002) method as previously described by Cerqueira et al. (2012). Briefly, the permeation cell was composed by two chambers divided by the film to be studied: the A/C PET (used as support film) or each type of multilayer. The permeation gas (oxygen) flowed continuously through the lower chamber, and nitrogen (as a carrier gas) was passed through upper chamber. The flow rate of oxygen and nitrogen was maintained constant and controlled by a gas flow meter (J and W Scientific, ADM 2000, USA) to keep its pressure constant in the compartment. The permeant gas passing across the film was swept by the carrier gas and analysed by gas chromatography (Chrompack 9001, Middelburg, Netherlands) at $110{ }^{\circ} \mathrm{C}$ with a column Porapak Q80/100 mesh $2 \mathrm{~m} \times 1 / 8$ in. $\times 2 \mathrm{~mm}$ SS equipped with a hydrogen flame ion detector. Helium (at $23 \mathrm{~mL} \mathrm{~min}^{-1}$ ) was used as carrier gas in the chromatograph. Calibration was done with a standard mixture containing $10 \% \mathrm{CO}_{2}, 20 \% \mathrm{O}_{2}$ and $70 \% \mathrm{~N}_{2}$. The oxygen permeation rate was determined when the steady state was reached and three replicates for oxygen permeability measurements were obtained for each sample.

The oxygen transmission rate $\left(\mathrm{O}_{2} \mathrm{TR}\right)$ of the polysaccharide or polysaccharide/protein nanolaminated coating (without A/C PET) was determined by the following equation (Cooksey et al. 1999).

$\mathrm{O}_{2} \mathrm{TR}_{B}=\frac{1}{\frac{1}{O_{2} \mathrm{TR}_{T}}-\frac{1}{O_{2} \mathrm{TR}_{A}}}$

where A, B and T refer to the sustaining layer (A/C PET), the polysaccharide-protein nanolaminated coating and the resulting nanolaminated film (A, B, C, D and E), respectively.

\section{Analysis}

\section{Fungal Strain}

Stock cultures of Alternaria sp. (MUM 02.42) and Rhizopus stolonifer (MUM 10.260) were supplied by Micoteca da 
Universidade do Minho (MUM, Braga, Portugal). Fungus were inoculated on Potato Dextrose Agar (PDA) and incubated at $25{ }^{\circ} \mathrm{C}$ until sporulation. The concentration of each inoculum was adjusted by means of a haemocytometer at $10^{4}$ spores per millilitre.

\section{Antifunfal Effectiveness of Nanolaminated Films}

Antimicrobial effectiveness of nanolaminated films was determined by adapting the methodology used by Kristo et al. (2008).

Aliquots of PDA $(7 \mathrm{~mL})$ infected by the fungus were poured into 50-mm Petri dishes. After solidification of the culture medium, the support films (A/C PET) and each multilayer system (A, B, C, D, E) of the same diameter as the Petri dishes $\left(5 \mathrm{~cm}^{2}\right)$ were placed on the surface. Inoculated uncoated PDA was used as control. Petri dishes were then covered with parafilm to avoid dehydration and stored at $25{ }^{\circ} \mathrm{C}$ for 30 days. Alternaria sp. and $R$. stolonifer counts on PDA plates were examined immediately after inoculation and periodically during the storage period. In order to do this, each sample with the agar was removed aseptically from Petri dishes and placed in a sterile plastic bag with $50 \mathrm{~mL}$ of tryptone phosphate water (Becton, Dickinson and Company Sparks, Le Pont de Claix, France) and homogenized for $5 \mathrm{~min}$ in a Stomacher blender (3500, Seward Medical, UK). Serial dilutions were made and then poured onto PDA-containing Petri dishes. These Petri dishes were incubated for 4 days at $25{ }^{\circ} \mathrm{C}$ for evaluation of Alternaria sp. and $R$. stolonifer growth and colonies were counted. Antifungal properties analyses were run in duplicate.

\section{Statistical Analysis}

Statistical analysis of data was performed through analysis of variance (ANOVA) using Statgraphics Plus for Windows 5.1 (Manugistics Corp., Rockville, MD). Fisher's least significant difference (LSD) procedure was also used.

\section{Results and Discussion}

\section{Preparation of Nanolaminated Films}

\section{Characterization of Polyelectrolyte Solutions}

PET samples were previously aminolysed and treated with $\mathrm{HCl}$ to charge positively the support surface (A/C PET). Thus, the first negatively charged surface layer (alginate layer) will interact with the support surface by electrostatic forces and then with the subsequent positively charged layer. In order to guarantee the electrostatic interaction with the A/C PET surface, the charge of each solution was confirmed by the determination of zeta potential ( $\zeta$-potential) (Table 2$)$. The $\zeta$-potential value for alginate was found to be $-60.4 \pm 4.2 \mathrm{mV}$ at $\mathrm{pH} 7.0$, lower and oppositely charged in comparison to that found for chitosan $(65.4 \pm 3.7 \mathrm{mV})$, chitosan-carvacrol emulsions $(67.6 \pm 2.2$ and $53.2 \pm 4.5 \mathrm{mV})$ and zein-carvacrol nanocapsules $(44.6 \pm 1.0 \mathrm{mV})$, all of them at $\mathrm{pH}$ 3.0. This condition was enough to support the electrostatic self-assembly and to build the five layers, as described in Table 1 . The negative $\zeta$ potential value for alginate can be explained by the free carboxyl groups present in its structure at $\mathrm{pH} 7.0$ (higher that its $\mathrm{p} K$ a that stands at 3.5) (Harnsilawat et al. 2006; Saravanan and Rao 2010). These values of $\zeta$-potential were similar to those found by Carneiro-da-Cunha et al. (2010) for alginate $(-62.13 \mathrm{mV}$ at $\mathrm{pH} 7.0)$. At $\mathrm{pH} 3.0$, the amino groups of chitosan are positively charged and the reduction of the electrical net charge (decrease in $\zeta$-potential) with the increase of carvacrol content (only noticeable at carvacrol contents of $0.6 \%$ ) could be explained by the electrostatic interactions between chitosan and the carvacrol compounds at the tested $\mathrm{pH}(\mathrm{pH}$ 3.0) (Sánchez-González et al. 2011). In fact, the $\zeta$-potential of carvacrol dispersed in distilled water (in the absence of chitosan) was $-21.5 \pm 1.5 \mathrm{mV}$ and this negative charge was associated with the presence of dissociable compounds in the carvacrol and with the adsorption of negative ions on the droplet surface. At this $\mathrm{pH}$, the adsorption of chitosan on the droplets of carvacrol results in positively charged dispersed particles. The positive charge of amino groups of chitosan could be partially neutralized through the interaction with some negatively charged groups of the carvacrol components when adsorbed on the oil droplet surface. This charge in the surface of dispersed particles was also found by incorporation of chitosan to oil-in-water emulsions with different types of surfactants (Hou et al. 2010). It is worth to note that the high value of $\zeta$-potential (higher than $+30 \mathrm{mV}$ ) implied a strong surface charge of the particles, guaranteeing the action of the repulsive forces among these, and thus, the stability of chitosancarvacrol emulsions was ensured by steric stabilization. Aqueous solutions containing zein-carvacrol nanocapsules were also positively charged $(44.6 \pm 1 \mathrm{mV})$, which also guaranteed the construction of nanolaminated films. This $\zeta$-potential value was higher than that reported by Zhang et al. (2014) for zein/thymol nanocapsules obtained by freeze-drying and redispersed in deionized water $(28.1 \pm 0.14 \mathrm{mV})$. The encapsulation efficiency of carvacrol in zein nanoparticles was $90.0 \pm 1.5 \%$ and is in agreement with results presented elsewhere (Luo et al. 2011).

The particle size distribution was also characterized and it was observed that chitosan-carvacrol solutions provided larger particle size diameters $(2.0 \pm 0.2$ and $4.5 \pm 0.1 \mu \mathrm{m}$ for those $\mathrm{CH}-0.09 \mathrm{C}$ and $\mathrm{CH}-0.6 \mathrm{C}$, respectively) than aqueous solutions of zein-carvacrol nanocapsules $(115 \pm 10 \mathrm{~nm})$. The differences in particle size can be explained by the solubility of each protein in the aqueous solution and thus by polymer-solvent 
Table $2 \zeta$-potential values of each polyelectrolyte solution assembly used for multilayer

\begin{tabular}{ll}
\hline Layer & $\zeta$-potential $(\mathrm{mV})$ \\
\hline Carvacrol oil (C) & $-21.5(1.2)^{\mathrm{a}}$ \\
$\mathrm{ALG}$ & $-60.4(4.2)^{\mathrm{b}}$ \\
$\mathrm{CH}$ & $65.4(3.7)^{\mathrm{c}}$ \\
$\mathrm{CH}-0.09 \mathrm{C}$ & $67.6(2.2)^{\mathrm{c}}$ \\
$\mathrm{CH}-0.6 \mathrm{C}$ & $53.2(4.5)^{\mathrm{d}}$ \\
Z/carv & $44.6(1.0)^{\mathrm{e}}$ \\
\hline a-d Different superscripts within the same \\
column indicate significant differences \\
among polyelectrolyte solutions $(p<0.05)$
\end{tabular}

interactions. It is well known that the use of a solvent for a particular polymer in which it is highly soluble (which is the case of chitosan in the aqueous-acid media, $\mathrm{pH}=3$ ), makes the polymer chains to swell and expand, favouring polymer/ solvent interactions, and thus, the particle size will increase. However, when a solvent of poor solubility for a particular polymer is used, polymer-polymer self-interactions preferentially occur (Luo et al. 2012). Therefore, the addition of zein-carvacrol in the antisolvent aqueous phase, which is a well-known procedure for nanoencapsulation ( $\mathrm{Li}$ et al. 2012), will favour intermolecular interactions and the formation of the nanoparticles.

\section{Characterization of the Coating Procedure of A/C PET}

Contact angle measurements are useful tools to determine the hydrophobic or hydrophilic nature of film's surface: lower values $\left(\theta<20^{\circ}\right)$ are closely related with wettable surfaces; on the contrary, the hydrophobic surfaces show high values $\left(\theta>70^{\circ}\right)$ of the contact angle. Thus, the deposition of successive layers onto A/C PET can be followed by contact angle measurements due to the different wettability properties of the electrolyte solutions used for nanolaminated films formation. Figure 1 displays contact angle values observed in A/C PET and when successive layers were added. Each data point is an average of seven determinations and the error bars represent the standard deviation. The original PET surface showed a contact angle value of $83.2 \pm 1.5^{\circ}$ which was significantly higher $(p<0.05)$ than the value of $77.0 \pm 1.0^{\circ}$ found for $\mathrm{A} / \mathrm{C}$ PET surface, indicating the hydrophobic nature of both $\left(\theta>70^{\circ}\right)$. This difference in the contact angle values confirmed the effectiveness of aminolysis of the original PET surface. Similar results were observed by Medeiros et al. (2012 and 2013), Carneiro-da-Cunha et al. (2010) and Xu et al. (2008) for aminolysed PET. The successful assembly of the polyelectrolytes on A/C PET films was verified by the alternating values of contact angles. It was observed that contact angles of alternate assembling of polycation layers were higher than those of polyanion layers (e.g. alginate), illustrating that alginate was more hydrophilic than both chitosan and

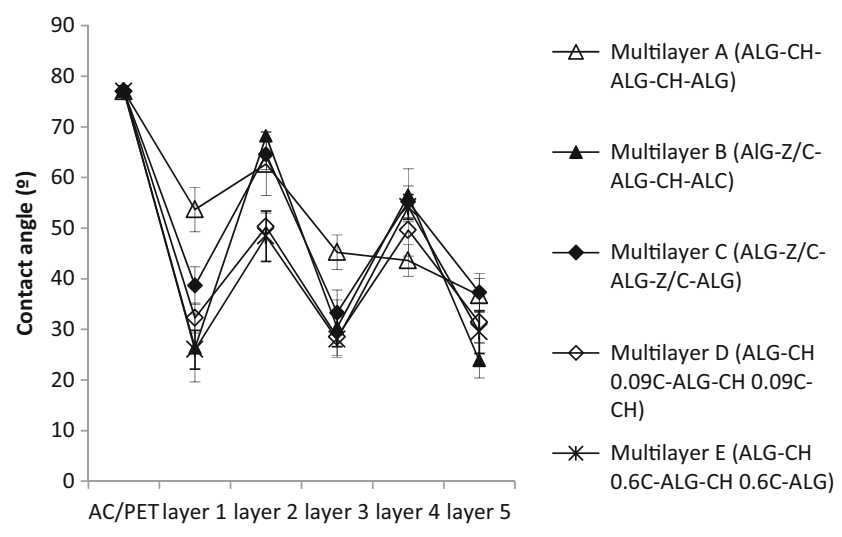

Fig. 1 Contact angle measured on original A/C PET and on the five successive layers of each nanolaminated film system

zein/carvacrol solutions. Comparing the polycation layers, the contact angles of adsorbed chitosan layers were much higher than adsorbed chitosan-carvacrol layers which implied a more hydrophobic structure of chitosan than the resulting chitosancarvacrol layers. A similar behaviour was observed by Kurek et al. (2014) in chitosan-carvacrol films.

\section{Water Vapour and Oxygen Transmission Rates of Nanolaminated Films}

Barrier properties are one of the most important issues to be considered in coating materials since the presence of oxygen could lead to the decrease in food shelf-life and quality ( $\mathrm{Li}$ et al. 2013). Water vapour (WVTR) and oxygen $\left(\mathrm{O}_{2} \mathrm{TR}\right)$ transmission rates of nanolaminated films were measured and the results are shown in Table 3. The WVTR values for the developed multilayer films were lower than those previously reported for neat alginate or chitosan films, $17 \times 10^{-3}$ and $15 \times 10^{-3} \mathrm{~g} \mathrm{~m}^{-2} \mathrm{~s}^{-1}$, respectively (Jost et al. 2014; Rodríguez-Núñez et al. 2014). The decrease in WVTR for multilayer films could be attributed to the strong interactions established between successive alginate and chitosan layers (Jang et al. 2008). The presence of carvacrol and zein nanocapsules greatly decreased the value of WVTR as compared to the multilayer system prepared with alginate and chitosan which may be due, on the one hand, to the hydrophobic nature of carvacrol and, on the other hand, to the better barrier properties of zein as compared to other proteins and polysaccharides (Cho, Park and Rhee 2002; Fabra, LópezRubio and Lagaron 2014).

The oxygen transmission rate behaved differently. The addition of carvacrol to the chitosan emulsion increased the value of $\mathrm{O}_{2} \mathrm{TR}$ which can be mainly attributed to its liquid state at room temperature, favouring molecular mobility and diffusion phenomena through the lipid phase. Thus, $\mathrm{O}_{2}$ can permeate easily through the films containing a higher amount of carvacrol (multilayer "E"). On the other hand, previous works (Fabra, Talens, Gavara and Chiralt 2012) reported that liquid 
Table 3 Water vapour and oxygen transmission rate of nanolaminated films and polysaccharide/protein multilayer

\begin{tabular}{llllll}
\hline Sample & \multicolumn{2}{l}{ WVTR $\cdot 10^{3}\left(\mathrm{~g} \mathrm{~m}^{-2} \mathrm{~s}^{-1}\right)$} & & & $\mathrm{O}_{2} \mathrm{TR} \cdot 10^{7}\left(\mathrm{~g} \mathrm{~m}^{-2} \mathrm{~s}^{-1}\right)$ \\
\cline { 2 - 3 } \cline { 5 - 6 } & Nanolaminated film & Polysaccharide/protein multilayer & & Nanolaminated film & Polysaccharide/protein multilayer \\
\hline AC/PET & $1.31(0.01)^{\mathrm{a}}$ & & & $11.71(0.40)^{\mathrm{a}}$ & \\
1 & $1.07(0.17)^{\mathrm{ab}}$ & 5.84 & & $6.50(0.48)^{\mathrm{b}}$ & 14.61 \\
$\mathrm{~A}$ & $0.92(0.09)^{\mathrm{b}}$ & 3.09 & & $6.51(0.65)^{\mathrm{b}}$ & 14.66 \\
$\mathrm{~B}$ & $0.85(0.06)^{\mathrm{b}}$ & 2.42 & & $10.30(0.60)^{\mathrm{b}}$ & 20.76 \\
$\mathrm{C}$ & $0.79(0.07)^{\mathrm{b}}$ & 1.99 & $10.70)^{\mathrm{a}}$ & 89.12 \\
$\mathrm{D}$ & $0.79(0.07)^{\mathrm{b}}$ & 1.99 & $10.75(0.81)^{\mathrm{a}}$ & 131.13 \\
\hline
\end{tabular}

${ }^{\mathrm{a}, \mathrm{b}}$ Different superscripts within the same column indicate significant differences among samples $(p<0.05)$

lipids such as oleic acid had a plasticizing effect on hydrocolloids matrices, which also helped to promote diffusion phenomena through the matrix, decreasing gas barrier efficiency. However, this did not occur when carvacrol was encapsulated within the zein matrix probably due to zein matrix acting as an oxygen barrier. In fact, zein has excellent potential as gas barrier in dry state (Fabra et al. 2013, 2014).

\section{Antifungal Activity of Nanolaminated Films}

The mechanism of the antifungal effect depends predominantly on the ability to affect the function of cellular lipoprotein membranes, causing an impairment of cellular ionic homeostasis, acidification of vacuolar and cytosolic $\mathrm{pH}$ and even the destruction of structural cellular integrity (Xu et al. 2008, Rao et al. 2010). In this work, the effect of active nanolaminated films against Alternaria sp. and $R$. stolonifer was analysed. The antifungal effect of nanolaminated films against Alternaria sp. was determined on PDA medium and is shown in Fig. 2. It is worth to note that, although some evaporation of carvacrol can occur during the preparation of chitosancarvacrol polyelectrolyte solutions and nanolaminated film formation, the initial carvacrol concentration in the filmforming solution was considered for the discussion. Results

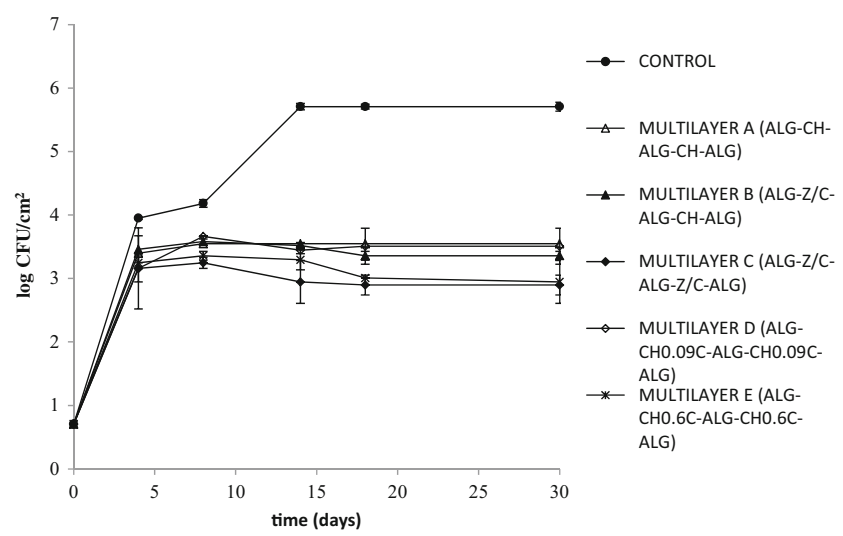

Fig. 2 Antifungal activity of the developed nanolaminated films against Alternaria sp. obtained for R. stolonifer cannot be shown since the growth of this fungus is highly invasive (Odeniyi, Onilude and Ayodele 2009) and the CFU could not be counted. In a recent work, García-Rincón et al. (2010) demonstrated that chitosan application to $R$. stolonifer also affected potassium efflux, $\mathrm{pH}$ of the media and $\mathrm{H}+$ ATP-ase of the plasma membrane activity and, thus, $R$. stolonifer survival.

Nanolaminated films were shown to have antifungal properties against Alternaria sp.; this happened even with those obtained without carvacrol, in this case most probably due to the presence of chitosan. Similarly, Bhaskara et al. (1998) reported that chitosan affected the spore viability of Alternaria alternata (a fungus typically found in tomato), whilst in other findings, the degree of inhibition of the growth and sporulation of mycelia from this fungus isolated from tomato fruits relied on the molecular weight of chitosan (Sánchez-Dominguez, BautistaBaños and Castillo-Ocampo 2007). From SEM (scanning electronic microscopy) images, these authors also observed that chitosan modified the mycelia morphology of $A$. alternata causing deformation, swollenness and nodulations. In a more recent work, Sanchez-Dominguez et al. (2011) confirmed that $A$. alternata is quite susceptible to chitosan treatments, demonstrating through TEM (transmission electronic microscopy) analysis that the cell wall of the fungal hyphae and conidia was seriously damaged. However, some fungi have been shown to be insensitive to chitosan activity. In this sense, Roller and Covill (1999) found, in similar studies, that the growth of Aspergillus flavus, Cladosporium cladosporioides and Penicillium aurantiogriseum was not affected by the presence of chitosan. In a more recent work, Sánchez-González et al. (2010) also showed that chitosan did not affect the growth of Penicillium italicum. Thus, the effectiveness of the antifungal activity of chitosan depends on the fungus.

As commented above, nanolaminated films containing carvacrol were prepared due to their antifungal properties. Rao et al. (2010) and Ahmad et al. (2011) reported that the molecular structure and relative position of the functional groups of carvacrol are responsible for the strong ability to dissolve and accumulate in the cell membrane, resulting in cell membrane 
destabilization which can be attributed to a more efficient disruption of proton transfer. In fact, Ultee et al. (2002) and Ahmad et al. (2011) pointed out that the antifungal properties of these compounds are connected simply to their ability to block ATP and ergosterol synthesis.

As can be observed in Fig. 2, the antifungal activity of nanolaminated films prepared by alternating layers of alginate with layers of nanocapsules of zein-carvacrol (multilayer C) was in the same range $(p>0.05)$ as nanolaminated films prepared with the highest content of carvacrol (multilayer E), probably due to the controlled release of the active agent (carvacrol) in the case of the multilayer $\mathrm{C}$ which delivers more efficiently the antifungal effect, avoiding the volatilization of this compound. No significant differences $(p>0.05)$ were found between nanolaminated films prepared with the lower amount of carvacrol $(0.09 \%, w / w)$ and those containing one layer of nanocapsules of zein-carvacrol (multilayer B). The volatile nature of carvacrol could contribute to the fact that the amount remaining in the multilayer with $0.09 \%(w / w)$ carvacrol will be too low to have an antifungal effect, showing no significant differences $(p>0.05)$ with those prepared without carvacrol (multilayer A).

Similar results were observed by Zabka and Pavela (2013), showing that carvacrol and thymol are the most promising antifungal agents against Fusarium oxysporum, Fusarium verticilioides, Penicillum brevicompactum, Penicillium expansum and A. flavus.

\section{Conclusion}

Nanolaminated coatings on a A/C PET support were successfully built-up through an alternate dipping procedure. The WVTR and $\mathrm{O}_{2}$ TR values for the developed multilayer films were lower than those previously reported for neat alginate or chitosan-based films. The presence of carvacrol and zein nanocapsules significantly decreased WVTR (up to $40 \%$ ) of nanolaminated films as compared to those prepared with alginate and chitosan. However, the oxygen barrier properties of nanolaminated films containing carvacrol were only improved (up to $45 \%$ ) when it was nanoencapsulated. Nanolaminated films prepared by alternating alginate with layers of nanocapsules of zein-carvacrol (multilayer $\mathrm{C}$ ) showed the highest antifungal activity against Alternaria $s p$., which did not significantly differ from nanolaminated films obtained with the highest amount of carvacrol (multilayer E), probably due to the controlled release of the active agent (carvacrol) in the case of the multilayer $\mathrm{C}$ which delivers more efficiently the active compound, avoiding its volatilization. Taking into account the overall properties of the developed nanolaminated films, those prepared with alternating layers of alginate and zein nanocapsules can be proposed as a good candidate to improve the shelf-life of foodstuffs due to the improved barrier properties and antifungal activity.
Acknowledgments The authors acknowledge financial support from FP7 IP project "ECOBIOCAP". M. J. Fabra is recipients of a Juan de la Cierva contract from the Spanish Ministry of Economy and Competitivity. Maria L. Flores-López thanks Mexican Science and Technology Council (CONACyT, Mexico) for PhD fellowship support (CONACyT Grant Number 215499/310847). The author Miguel A. Cerqueira is a recipient of a fellowship (SFRH/BPD/72753/2010) supported by Fundação para a Ciência e Tecnologia, POPH-QREN and FSE (FCT, Portugal). The authors also thank the FCT Strategic Project of UID/ BIO/04469/2013 unit, the project RECI/BBB-EBI/0179/2012 (FCOMP01-0124-FEDER-027462) and the project "BioInd - Biotechnology and Bioengineering for improved Industrial and Agro-Food processes," REF. NORTE-07-0124-FEDER-000028 Co-funded by the Programa Operacional Regional do Norte (ON.2-O Novo Norte), QREN, FEDER. The support of EU Cost Action FA0904 is gratefully acknowledged.

\section{References}

Ahmad, A., Khan, A., Akhtar, F., Yousuf, S., Xess, I., Khan, L. A., \& Manzoor, N. (2011). Fungicidal activity of thymol and carvacrol by disrupting ergosterol biosynthesis and membrane integrity against Candida. European Journal of Clinical Microbiology and Infectious Diseases, 30(1), 41-50.

ASTM (2002). D 3985-02 standard test method for oxygen gas transmission rate through plastic film and sheeting using a coulometric sensor. In ASTM Book of Standards, 15, 09.

ASTM (2011). Standard test methods for water vapour transmission of materials. Standard designations: annual book of ASTM standards. Philadelphia, PA: American Society for Testing and Materials.

Ben Arfa, A., Combes, S., Preziosi-Belloy, L., Gontard, N., \& Chalier, P. (2006). Antimicrobial activity of carvacrol related to its chemical structure. Letters in Applied Microbiology, 43, 149-154.

Bhaskara, M. V., AitBarka, E., Castaigne, F., \& Arul, J. (1998). Effect of chitosan on growth and toxin production by Alternaria alternate $f$. Sp. Lycopersici. Biocontrol Science and Technology, 8, 33-43.

Burt, S. (2004). Essential oils: their antibacterial properties and potential applications in foods - a review. International Journal of Food Microbiology, 94(3), 223-253.

Carneiro-da-Cunha, M. G., Cerqueira, M. A., Souza, B. W. S., Carvalho, S., Quintas, M. A. C., Teixeira, J. A., \& Vicente, A. A. (2010). Physical and termal properties of a chitosan/alginate nanolayered PET film. Carbohydrate Polymers, 82, 153-159.

Casariego, A., Souza, B. W. S., Cerqueira, M. A., Teixeira, J. A., Cruz, L., Díaz, R., \& Vicente, A. A. (2009). Chitosan/clay films' properties as affected by biopolymer and clay micro/nanoparticles' concentrations. Food Hydrocolloids, 23, 1895-1902.

Cerqueira, M. A., Souza, B. W. S., Teixeira, J. A., \& Vicente, A. A. (2012). Effects of interactions between the constituents of chitosan-edible films on their physical properties. Food and Bioprocess Technology, 5(8), 3181-3192.

Cho, S. Y., Park, J. W., \& Rhee, C. (2002). Properties of laminated films from whey powder and sodium caseinate mixtures and zein layers. Food Science and Technology., 35, 135-139.

Cooksey, K., Marsh, K. S., \& Doar, L. H. (1999). Predicting permeability and transmission rate for multilayer materials. Food Technology, 53(9), 60-63.

Decher, G., and Schlenoff, J.B. (2003). Multilayer thin films: sequential assembly of nanocomposite materials. Weinheim, Germany:WileyVCH. p 543

Fabra, M. J., Talens, P., Gavara, R., \& Chiralt, A. (2012). Barrier properties of sodium caseinate films as affected by lipid composition and moisture content. Journal of Food Engineering, 109, 372-379. 
Fabra, M. J., López-Rubio, A., \& Lagaron, J. M. (2013). High barrier polyhydroxyalcanoate food packaging film by means of nanostructured electrospun interlayers of zein prolamine. Food Hydrocolloids, $32,106-114$

Fabra, M. J., López-Rubio, A., \& Lagaron, J. M. (2014). Nanostructured interlayers of zein to improve the barrier properties of high barrier polyhydroxyalkanoates and other polyesters. Journal of Food Engineering, 127, 1-9.

Fu, J., Ji, J., Yuan, W., \& Shen, J. (2005). Construction of anti-adhesive and antibacterial multilayer films via layer-by-layer assembly of heparin and chitosan. Biomaterials, 26, 6684-6692.

García-Rincón, J., Vega-Pérez, J., Guerra-Sánchez, M. G., HernándezLauzardo, A. N., Peña-Díaz, A., \& Velázquez-Del Valle, M. G. (2010). Effect of chitosan on growth and plasma membrane properties of Rhizopus stolonifer (Ehrenb.:Fr.) Vuill. Pesticide Biochemistry and Physiology, 97(3), 275-278.

Harnsilawat, T., Pongsawatmanit, R., \& McClements, D. J. (2006). Characterization of $\beta$-lactoglobulin-sodium alginate interactions in aqueous solutions: a calorimetry, light scattering, electrophoretic mobility and solubility study. Food Hydrocolloids, 20, 577-585.

Hinrichsen, G., Hoffmann, A., Schleeh, T., \& Macht, C. (2003). Continuous production of ultrathin polymeric nanofilms using the spontaneous film formation technique. Advances in Polymer Technology, 22(2), 120-125.

Hou, Z., Gao, Y., Yuan, F., Liu, Y., Li, C., \& Xu, D. (2010). Investigation into the physicochemical stability and rheological properties of betacarotene emulsion stabilized by soybean soluble polysaccharides and chitosan. Journal of Agriculture and Food Chemistry, 58(15), 8604-8611.

Jang, W.-S., Rawson, I., \& Grunlan, J. C. (2008). Layer-by-layer assembly of thin film oxygen barrier. Thin Solid Films, 516(15), 48194826.

Jost, V., Kobsik, K., Schmid, M., \& Noller, K. (2014). Influence of plasticizer on the barrier, mechanical and grease resistance properties of alginate cast films. Carbohydrate Polymers, 309-319.

Kristo, E., Koutsoumanis, K. P., \& Biliaderis, C. G. (2008). Thermal, mechanical and water vapor barrier properties of sodium caseinate films containing antimicrobials and their inhibitory action on Listeria monocytogenes. Food Hydrocolloids, 22, 373-386.

Kurek, M., Guinault, A., Voilley, A., Galic, K., \& Debeaufort, F. (2014). Effect of relative humidity on carvacrol release and permeation properties of chitosan based films and coatings. Food Chemistry, 144, 9-17.

Lambert, R. J. W., Skandamis, P. N., Coote, P. J., \& Nychas, G. J. E. (2001). A study of the minimum inhibitory concentration and mode of action of oregano essential oil, thymol and carvacrol. Journal of Applied Microbiology, 91(3), 453-462.

Li, K. K., Yin, S. W., Yang, X. Q., Tang, C. H., \& Wei, Z. H. (2012). Fabrication and characterization of novel antimicrobial films derived from thymol-loaded zein-sodium caseinate (SC) nanoparticles. Journal of Agricultural and Food Chemistry, 60, 11592-11600.

Li, C., Huang, W. Y., Wang, X. N., \& Liu, W. X. (2013). Oxygen radical absorbance capacity of different varieties of strawberry and the antioxidant stability in storage. Molecules, 18(2), 1528-1539.

Luo, Y. C., Zhang, B., Whent, M., Yu, L. L., \& Wang, Q. (2011). Preparation and characterization of zein/chitosan complex for encapsulation of alpha-tocopherol, and its in vitro controlled release study. Colloids and Surfurce B: Biointerfaces, 85(2), 145-152.

Luo, C. J., Stride, E., \& Edirisinghe, M. (2012). Mapping the influence of solubility and dielectric constant on electrospinning polycaprolactone solutions. Macromolecules, 45(11), 4669-4680.

McHugh, T. H., Avena-Bustillos, R. J., \& Krochta, J. M. (1993). Hydrophilic edible film: modified procedure for water vapor permeability and explanation of thickness effects. Journal Food Science, 58(4), 899-903.

Medeiros, B. G. S., Pinheiro, A. C., Teixeira, J. A., Vicente, A. A., \& Carneiro-da-Cunha, M. G. (2012). Polysaccharide/protein nanomultilayer coatings: construction, characterization and evaluation of their effect on 'Rocha' pear (Pyrus communis L.) shelf-life. Food and Bioprocess Technology, 5, 2435-2445.

Medeiros, B. G. S., Souza, M. P., Pinheiro, A. C., Bourbon, A. I., Cerqueira, M. A., Vicente, A. A., \& Carneiro-da-Cunha, M. G. (2013). Physical characterisation of an alginate/lysozyme nanolaminate coating and its evaluation on 'coalho' cheese shelf life. Food and Bioprocess Technology, 7(4), 1088-1098.

Newman, A. W., \& Kwok, D. Y. (1999). Contact angle measurement and contact angle interpretation. Advances in Colloid andInterface Science, 81, 167-249.

Nostro, A., \& Papalia, T. (2012). Antimicrobial activity of carvacrol: current progress and future prospective. Recent Patents on AntiInfective Drug Discovery, 7(1), 28-35.

Odeniyi, O. A., Onilude, A. A., \& Ayodele, M. A. (2009). Growth and substrate utilization patterns of a Rhizopus stolonifer strain isolated from depolymerizing rice husk. World Applied Sciences Journal, 6(5), 595-599.

Patel, A. R., Bouwens, E. C., \& Velikov, K. P. (2010). Sodium caseinate stabilized zein colloidal particles. Journal of Agriculture and Food Chemistry, 58(23), 12497-12503.

Peng, J. B., Barnes, G. T., \& Gentle, I. R. (2001). The structures of langmuir-blodgett films of fatty acids and their salts. Advances in Colloid and Interface Science, 91, 163-219.

Pinheiro, A. C., Bourbon, A. I., Medeiros, B. G. D. S., Da Silva, L. H. M., Da Silve, M. C. H., Carneiro-da-Cunha, M. G., Coimbra, M. A., \& Vicente, A. A. (2012). Interactions between K-carrageenan and chitosan in nanolayered coatings - structural and transport properties. Carbohydrate Polymers, 1081-1090.

Rao, A., Zhang, Y. Q., Muend, S., \& Rao, R. (2010). Mechanism of antifungal activity of terpenoid phenols resembles calcium stress and inhibition of the TOR pathway. Antimicrobial Agents Chemotherafy, 54(12), 5062-5069.

Rodríguez-Núñez, J. R., Madera-Santana, T. J., Sánchez-Machado, D. I., López-Cervantes, J., \& Soto Valdez, H. (2014). Chitosan/ hydrophilic plasticizer-based films: preparation, physicochemical and antimicrobial properties. Journal of Polymers and the Environment, 22(1), 41-51.

Roller, S., \& Covill, N. (1999). The antifungal properties of chitosan in laboratory media and apple juice. International Journal of Food Microbiology, 47, 67-77.

Sánchez-Dominguez, D., Bautista-Baños, S., \& Castillo-Ocampo, P. (2007). Efecto del quitosano en el desarrollo y morfología de Alternaria alternata (Fr) Keissl. Anales de Biología, 29, 23-32.

Sánchez-Dominguez, D., Rios, M. Y., Castillo-Ocampo, P., ZavalaPadilla, G., Ramos-García, M., \& Bautista-Baños, S. (2011). Cytological and biochemical changes induced by chitosan in the pathosystem Alternaria alternata-tomato. Pesticide Biochemistry and Physiology, 99, 250-255.

Sánchez-González, L., Cháfer, M., Chiralt, A., \& González-Martínez, C. (2010). Physical properties of edible chitosan films containing bergamot essential oil and heir inhibitory action on Penicillium italicum. Carbohydrate Polymer, 82, 277-283.

Sánchez-González, L., Chiralt, A., González-Martínez, C., \& Cháfer, M. (2011). Effect of essential oils on properties of film forming emulsions and films base don hydroxypropylmethylcellulose and chitosan. Journal of Food Engineering, 98, 443-452.

Saravanan, M., \& Rao, K. P. (2010). Pectin-gelatin and alginate-gelatin complex coacervation for controlled drug delivery: influence of anionic polysaccharides and drugs being encapsulated on physicochemical properties of microcapsules. Carbohydrate Polymers, 80(3), 808-816.

Ultee, A., Bennik, M. H. J., \& Moezelaar, R. (2002). The phenolic hydroxyl group of carvacrol is essential for action against the foodborne pathogen Bacillus cereus. Applied and Environmental Microbiolgy, 68(4), 1561-1568. 
Weis, J., Takhistow, P., \& McClements, J. (2006). Functional materials on food nanotechnology. Journal of Food Science, 9, 107-116.

Xu, J., Zhou, F., Ji, B. P., Pei, R. S., \& Xu, N. (2008). The antibacterial mechanism of carvacrol and thymol against Escherichia coli. Letter of Applied Microbiology, 47(3), 174-179.

Zabka, M., \& Pavela, R. (2013). Antifungal efficacy of some natural phenolic compounds against significant pathogenic and toxinogenic filamentous fungi. Chemosphere, 93, 1051-1056.

Zhang, Y., Niu, Y., Luo, Y., Ge, M., Yang, T., \& Yu, L. (2014). Fabrication, characterization and antimicrobial activities of thymol loaded zein nanoparticles stabilized by sodium caseinate-chitosan hydrochloride double layers. Food Chemistry, 142, 269-275.

Zhong, Y., Li, Y., \& Haynie, D. (2006). Control of stability of polypeptide multilayer nanofilms by quantitative control of disulfide bond formation. Nanotechnology, 17, 5726-5734.

Zhong, Q., Tian, H., \& Zivanovic, S. (2009). Encapsulation of fish oil in solid zein particles by liquid-liquid dispersion. Journal of Food Processing and Preservation, 33(2), 255-270. 